\title{
Гальваномагнитные свойства моносилицида кобальта и сплавов на его основе
}

\author{
(C) А.Ю. Овчинников ${ }^{1,2}$, П.П. Константинов ${ }^{1}$, Д.А. Пшенай-Северин ${ }^{1,2}$, А.Т. Бурков ${ }^{1}$ \\ ${ }^{1}$ Физико-технический институт им. А.Ф. Иоффе Российской академии наук, \\ 194021 Санкт-Петербург, Россия \\ ${ }^{2}$ Санкт-Петербургский политехнический университет Петра Великого, \\ 195251 Санкт-Петербург, Россия \\ E-mail: ovchinnikov.ayu@yandex.ru
}

Поступила в Редакцию 7 февраля 2019 г.

В окончательной редакции 10 февраля 2019 г.

Принята к публикации 14 февраля 2019 г.

Исследованы коэффициент Холла и проводимость моносилицида кобальта $\mathrm{CoSi}$, а также сплавов $\mathrm{Co}_{1-x} \mathrm{Fe}_{x} \mathrm{Si}$ и $\mathrm{Co}_{1-x} \mathrm{Ni}_{x} \mathrm{Co}$ с содержанием до 8 aт\% железа и до 5 aт\% никеля. Измерены температурные зависимости коэффициента Холла и проводимости в диапазоне температур 77-800 К. Теоретическая интерпретация экспериментальных зависимостей выполнена на основе двух разных моделей электронной структуры соединения: простой 2-зонной полуметаллической структуры с небольшим перекрытием изотропных параболических зон и электронной структуры, рассчитанной из первых принципов и содержащей вблизи энергии Ферми топологические особенности с многократно вырожденными пересечениями зон.

DOI: $10.21883 /$ FTP.2019.06.47720.29

\section{1. Введение}

Моносилицид кобальта $(\mathrm{CoSi})$, а также его твердые растворы с замещением атомов кобальта на никель $(\mathrm{Ni})$ и железо $(\mathrm{Fe})$ рассматривались как перспективные термоэлектрические материалы еще в 60-х годах прошлого века $[1,2]$. Эти материалы достаточно просто синтезировать, они значительно дешевле традиционного теллурида висмута, устойчивы при высоких температурах, механически прочны, а стехиометрический CoSi обладает термоэдс порядка -80 мкВ/К и проводимостью до $10^{4}(\mathrm{OM} \cdot \mathrm{cm})^{-1}$ при комнатной температуре. С появлением новых сведений о зонной структуре этих соединений [3-5] вновь возник интерес к их исследованию.

В исследованиях $[3,4]$ был произведен расчет зонной структуры CoSi из первых принципов, а в работе [5] были изучены топологические свойства зонной структуры CoSi. B результате исследований было выявлено наличие особенностей электронного спектра, которые не учитываются в простой полуметаллической модели. Главной особенностью, потребовавшей модификации ранее использованных методов описания транспортных свойств, является наличие вблизи уровня Ферми зон с линейным законом дисперсии. В $Г$ и $R$ точках зоны Бриллюэна были обнаружены узлы с многократным вырождением зон, обладающие топологическим зарядом больше единицы. Поэтому данные материалы можно отнести к классу топологических полуметаллов.

В работе для расчета кинетических коэффициентов используются две модели зонной структуры. В первой модели, традиционной для полуметаллов, рассматривались две параболические зоны электронов и дырок с небольшим энергетическим перекрытием и учитывалось рассеяние носителей тока на акустических фононах [2]. В настоящей работе с использованием эксперименталь- ных температурных зависимостей электропроводности и коэффициента Холла получены параметры модели, обсуждаются ее особенности и определен диапазон температур, в которых достигается хорошее согласие расчета с экспериментом. Во второй модели мы использовали электронный спектр, полученный с помощью первопринципных расчетов. Коэффициент Холла $R_{\mathrm{H}}$ и проводимость $\sigma$ рассчитаны в приближении постоянного времени релаксации, часто встречающемся в литературе. Кроме того, был проведен также расчет с учетом зависимости времени релаксации от энергии, которая соответствовала рассеянию на фононах и близкодействующем потенциале точечных дефектов. Было проведено сравнение результатов расчета с использованием указанных приближений и показано, что наряду с особенностями зонной структуры для правильного описания кинетических коэффициентов важен также учет энергетической зависимости времени релаксации.

\section{2. Методика эксперимента}

Исследованные образцы являются монокристаллами. $\mathrm{CoSi}$ и твердые растворы $\mathrm{Co}_{1-x} \mathrm{Fe}_{x} \mathrm{Si}$ и $\mathrm{Co}_{1-x} \mathrm{Ni}_{x} \mathrm{Si}$ получены прямым сплавлением компонентов. После охлаждения материалы были раздроблены, а затем дополнительно протянуты по методу Бриджмена для рекристаллизации.

Коэффициент Холла и проводимость образцов измерены при температурах от 77 до $800 \mathrm{~K}$. При проведении исследований применялся двухчастотный метод измерения коэффициента Холла совместно с методом автоматической компенсации. В качестве источника компенсирующего напряжения используется напряжение от умножителя, перемножающего сигналы, пропорциональные току через образец и магнитному полю. Этот 
метод устраняет необходимость в стабилизации тока и магнитного поля в образце, что облегчает автоматизацию процедуры измерения. Максимальная сила тока, протекающего через образец, достигает 1 А, частота 72 Гц, амплитуда магнитного поля - 0.15 Тл, частота 50 Гц. Измерения холловского напряжения проводятся на разностной частоте 22 Гц. Чувствительность системы составляет $5 \cdot 10^{-10}$ В. Одновременно с постоянной Холла измеряется электрическое сопротивление образца. Подробное описание измерительной установки содержится в работе [6].

\section{3. Экспериментальные результаты и их обсуждение}

На рис. 1, 2 показаны зависимости коэффициента Холла $R_{\mathrm{H}}(T)$ и электропроводности $\sigma(T) \mathrm{CoSi}$ и соединений на его основе, измеренные в температурном диапазоне 77-800 K, а также расчетные кривые.
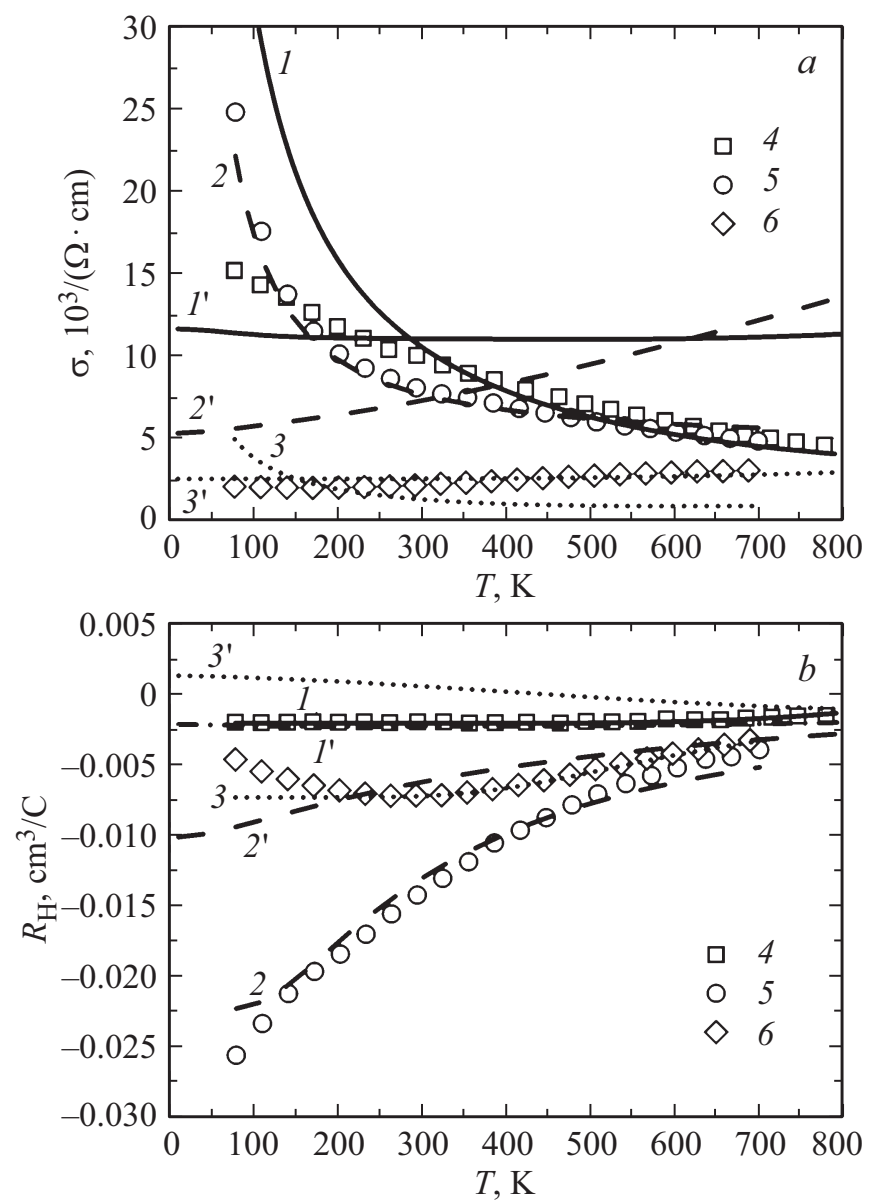

Рис. 1. Температурные зависимости электропроводности $\sigma(a)$ и коэффициента Холла $R_{\mathrm{H}}(b)$. Линии - результат расчета с использованием двузонной модели $[2,7](1-3)$ и первопринципных расчетов в приближении постоянного времени релаксации $\left(1^{\prime}-3^{\prime}\right)$. Символами обозначены экспериментальные значения. $\mathrm{Co}_{0.95} \mathrm{Ni}_{0.05} \mathrm{Si}-1,1^{\prime}, 4 ; \mathrm{CoSi}-2,2^{\prime}, 5$; $\mathrm{Co}_{0.96} \mathrm{Fe}_{0.04} \mathrm{Si}-3,3^{\prime}, 6$.
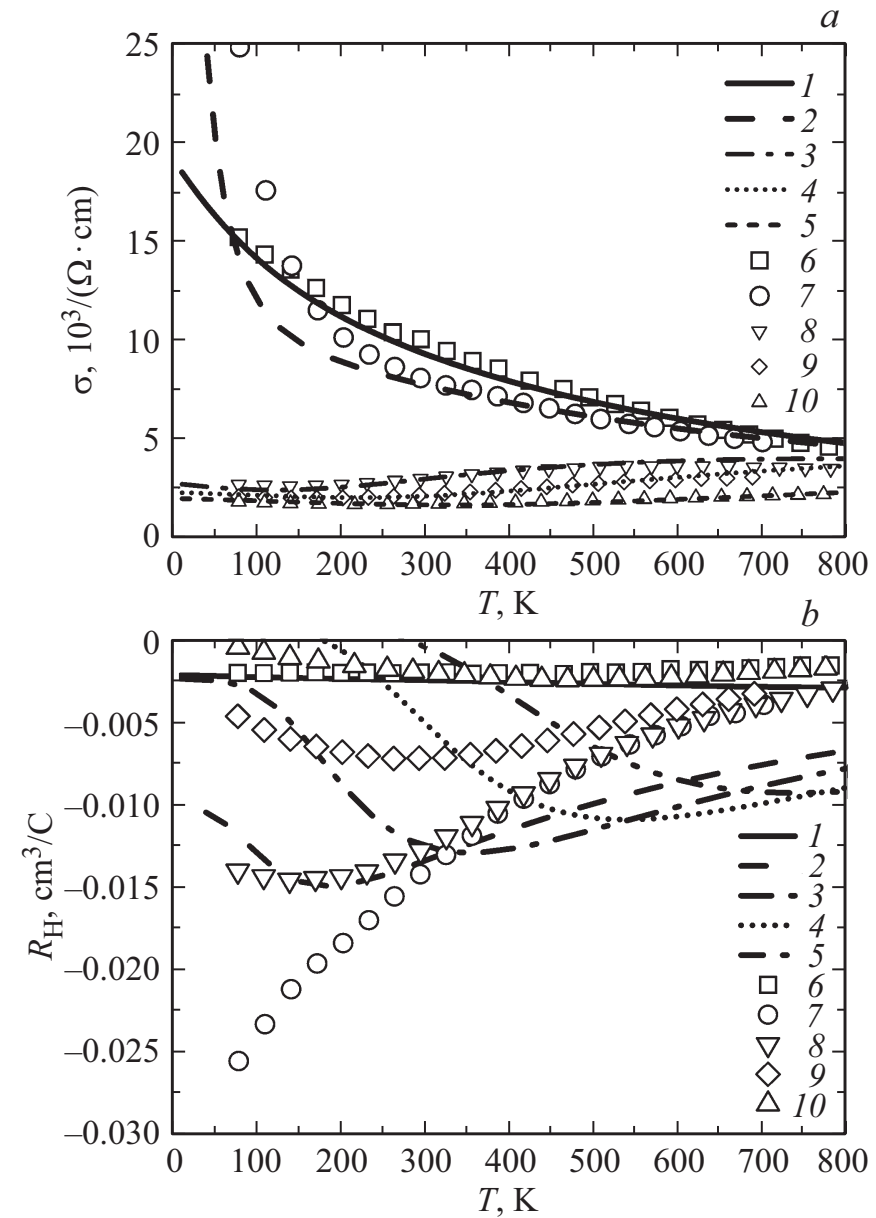

Рис. 2. Температурные зависимости $\sigma(a)$ и $R_{\mathrm{H}}(b)$, полученные с использованием первопринципных расчетов электронного спектра в приближении времени релаксации, зависящего от плотности состояний $(1-5)$. Символами обозначены экспериментальные значения. $\mathrm{Co}_{0.95} \mathrm{Ni}_{0.05} \mathrm{Si}-1,6$; $\mathrm{CoSi}$ - 2, 7; $\mathrm{Co}_{0.985} \mathrm{Fe}_{0.015} \mathrm{Si}-3,8 ; \mathrm{Co}_{0.96} \mathrm{Fe}_{0.04} \mathrm{Si}-4$, 9; $\mathrm{Co}_{0.92} \mathrm{Fe}_{0.08} \mathrm{Si}-5,10$.

Формулы для вычисления кинетических коэффициентов в двузонной параболической модели подробно обсуждались в работах [2,7]. Вид энергетического спектра схематически изображен на рис. 3 (пунктирная линия). Он описывается величинами эффективных масс электронов и дырок $\left(m_{n}, m_{p}\right)$, а также величиной энергетического перекрытия зоны проводимости и валентной зоны $\Delta E(T)$. В модели рассматривается рассеяние электронов на акустических фононах. Обратное время релаксации имеет следующую зависимость от температуры и энергии: $\tau^{-1} \sim T \varepsilon^{1 / 2}$. Отношение подвижностей электронов и дырок $\left(u_{n} / u_{p}\right)$ не зависит от температуры явно, а его величина определяется параметром $b$ :

$$
\frac{u_{n}}{u_{p}}=b \frac{F_{0}\left(\mu_{n}^{*}\right) / F_{1 / 2}\left(\mu_{n}^{*}\right)}{F_{0}\left(\mu_{p}^{*}\right) / F_{1 / 2}\left(\mu_{p}^{*}\right)},
$$

где $F_{i}\left(\mu^{*}\right)$ - интегралы Ферми, $\mu_{n}^{*}, \mu_{p}^{*}$ - приведенные химические потенциалы электронов и дырок 


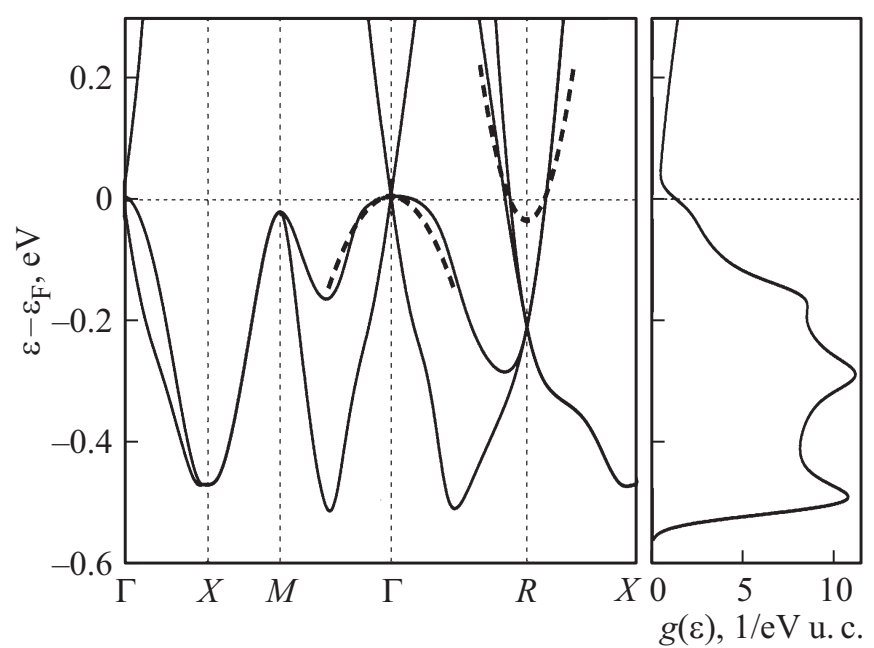

Рис. 3. Зонная структура (слева) и плотность состояний (справа), рассчитанные по методу функционала электронной плотности в данной работе. Пунктирными линиями показана электронная структура в двузонной полуметаллической модели [7].

в единицах $k_{0} T$, связанные соотношением $\mu_{n}^{*}+\mu_{p}^{*}=$ $=\Delta E(T) / k_{0} T$. В данной модели зависимость энергетического перекрытия зон от температуры учитывалась в виде $\Delta E(T)=\Delta E+\beta T$, где $\Delta E-$ величина перекрытия зон при нулевой температуре, а $\beta-$ ее линейный температурный коэффициент.

Положение уровня химического потенциала определялось независящей от температуры разностью концентраций электронов и дырок $(n-p)$, которые определялись соотношением

$$
n, p=4 \pi\left(\frac{2 m_{n, p}^{*} k_{0} T}{h^{2}}\right)^{3 / 2} F_{1 / 2}\left(\mu_{n, p}^{*}\right) .
$$

Коэффициент Холла вычислялся по формуле

$$
R_{\mathrm{H}}=\frac{p-n b^{2}}{e(p+n b)^{2}} .
$$

На рис. 1 приведены экспериментальные температурные зависимости электропроводности и коэффициента Холла для образцов $\mathrm{Co}_{0.95} \mathrm{Ni}_{0.05} \mathrm{Si}, \mathrm{CoSi}$ и $\mathrm{Co}_{0.96} \mathrm{Fe}_{0.04} \mathrm{Si}$. Соответствующие расчетные кривые 1-3 построены с использованием обсуждаемой модели. Эффективные массы для всех составов одинаковы и составляют $2 m_{0}$ и $6 m_{0}$ для электронов и дырок соответственно [7]. Значения остальных параметров были найдены из условия наилучшего согласия расчетных и экспериментальных температурных зависимостей коэффициента Холла и электропроводности, они приведены в таблице.

Из рис. 1 видно, что температурная зависимость электропроводности $\mathrm{CoSi}$ достаточно хорошо описывается теоретической кривой с учетом акустического рассеяния носителей тока. В образцах с замещением кобальта на никель при температурах ниже комнатной отличие расчетных и экспериментальных зависимостей электропроводности связано с влиянием сплавного рассеяния в твердых растворах, не учитываемого в модели [2,7]. Проводимость образцов с добавлением железа убывает в области низких температур и начинает возрастать при температурах выше 200-300 K, что не удается объяснить с точки зрения традиционной модели с энергетическим перекрытием параболических зон электронов и дырок.

Коэффициент Холла в образцах с никелем мал по модулю и практически не зависит от температуры. Это стандартное поведение хорошо описывается в данной модели. Однако в образце $\mathrm{CoSi}$ и в твердом растворе с железом имеется сильная температурная зависимость $R_{\mathrm{H}}(T)$, свидетельствующая о многозонной структуре электронного спектра. Она воспроизводится в расчетах при температурах выше комнатной. В твердых pactворах с железом коэффициент Холла возрастает по модулю при низких температурах, имеет максимум при $200 \mathrm{~K}$ для образца с $1.5 \mathrm{aT} \% \mathrm{Fe}$. С увеличением содержания $\mathrm{Fe}$ в твердом растворе возрастает температуpa, при которой наблюдается максимум: при содержании 4 ат\% Fе максимум наблюдается при температуре $300 \mathrm{~K}$, а при содержании $8 \mathrm{aT} \% \mathrm{Fe}$ - при $500 \mathrm{~K}$. При дальнейшем росте температуры абсолютное значение $R_{\mathrm{H}}$ уменьшается. В то же время расчетные кривые $R_{\mathrm{H}}(T)$ при низких температурах выходят на насыщение.

Как показало сравнение с данными работ [2,7], полученный набор параметров не является единственным. Это связано с тем, что кинетические коэффициенты одновременно определяются видом энергетических зависимостей как электронного спектра, так и времени релаксации, вклад которых в рамках данной модели оказывается невозможным разделить.

Для того чтобы уменьшить эту неопределенность, а также учесть особенности сложного электронного спектра $\mathrm{CoSi}$, во второй модели для описания транспортных свойств использовались результаты первопринципного расчета [3-5]. Расчет энергетического спектра проводился по методу функционала электронной плотности в пакете QuantumEspresso [8] с использованием градиентного приближения для функционала электронной плотности без учета спин-орбитального взаимодействия. Число плоских волн определялось максимальной энергией 90Ry, для интегрирования по зоне Бриллюэна использовалась сетка Монхорста-Пака $6 \times 6 \times 6$. Равновесные параметры решетки оказались равны $a=4.4348 \AA, x_{\mathrm{Co}}=0.144, x_{\mathrm{Si}}=0.843$. Вид зонной структуры приведен на рис. 3 (сплошные кривые).

Параметры двузонной модели для описания свойств твердых растворов на основе моносилицида кобальта

\begin{tabular}{l|c|c|c|c}
\hline \multicolumn{1}{c|}{ Состав } & $n-p, 10^{20} \mathrm{~cm}^{-3}$ & $\Delta E$, мэВ & $\beta, 10^{-6}{ }_{\ni} \mathrm{B} / \mathrm{K}$ & $b$ \\
\hline $\mathrm{Co}_{0.95} \mathrm{Ni}_{0.05} \mathrm{Si}$ & 30 & -30 & 20 & 3.5 \\
$\mathrm{CoSi}$ & 2.8 & -41 & 90 & 6.8 \\
$\mathrm{Co}_{0.96} \mathrm{Fe}_{0.04} \mathrm{Si}$ & -8.5 & 0 & 150 & 8.4
\end{tabular}


Для вычисления кинетических коэффициентов необходимы значения одноэлектронных энергий на более плотной сетке в зоне Бриллюэна $(64 \times 64 \times 64)$, которые были получены с использованием ваннье-интерполяции зонной структуры [9].

В этой модели были использованы два приближения для времени релаксации электронов: постоянное время релаксации и время релаксации, обратно пропорциональное плотности состояний. Первое приближение наиболее часто используется в литературе для расчета кинетических коэффициентов с использованием первопринципного подхода. Второе приближение для энергетической зависимости времени релаксации позволяет описать основные черты рассеяния носителей тока на акустических фононах и на близкодействующем потенциале точечных дефектов (сплавное рассеяние в твердых растворах):

$$
\tau \propto \frac{1}{\left(w_{a} T+w_{d}\right) g(\varepsilon)},
$$

где $g(\varepsilon)$ - плотность состояний, а параметры $w_{a}$ и $w_{d}$ определяют интенсивность акустического и сплавного рассеяния соответственно.

Формулы для вычисления электропроводности приведены в работе [9]:

$$
\sigma_{i j}=e^{2} \int_{-\infty}^{+\infty}\left(-\frac{\partial f_{0}(\varepsilon, \mu, T)}{\partial \varepsilon}\right) \Theta_{i j}(\varepsilon) d \varepsilon,
$$

где $f_{0}(\varepsilon, \mu, T)$ - функция распределения Ферми, $\Theta_{i j}(\varepsilon)$ - транспортная функция распределения:

$$
\Theta_{i j}(\varepsilon)=\frac{2}{V} \sum_{n, \mathbf{k}} v_{i}(n, \mathbf{k}) v_{j}(n, \mathbf{k}) \tau(\varepsilon) \delta\left(\varepsilon-\varepsilon_{n, \mathbf{k}}\right),
$$

a $v_{i}(n, \mathbf{k})=\hbar^{-1} \partial \varepsilon_{n, \mathbf{k}} / \partial k_{i}$ - компоненты скорости электрона с волновым вектором $\mathbf{k}$ в зоне с номером $n$.

Выражения для коэффициента Холла в кубическом кристалле были получены из общих формул [10] в приближении времени релаксации, зависящего от энергии:

$$
R=\frac{\sigma_{123}}{\sigma_{11}^{2}}
$$

где

$$
\sigma_{123}=-\frac{e^{3}}{c} \int_{-\infty}^{+\infty}\left(-\frac{\partial f_{0}(\varepsilon, \mu, T)}{\partial \varepsilon}\right) \Theta_{R_{\mathrm{H}}}(\varepsilon) d \varepsilon
$$

а входящая в него функция распределения

$$
\begin{aligned}
\Theta_{R_{\mathrm{H}}}(\varepsilon) & =\frac{2}{V} \sum_{n, \mathbf{k}}\left(v_{2}^{2}(n, \mathbf{k}) m_{11}^{-1}(n, \mathbf{k})\right. \\
& \left.-v_{1}(n, \mathbf{k}) v_{2}(n, \mathbf{k}) m_{12}^{-1}(n, \mathbf{k})\right) \tau^{2}(\varepsilon) \delta\left(\varepsilon-\varepsilon_{n, \mathbf{k}}\right),
\end{aligned}
$$

где $m_{i j}^{-1}(n, \mathbf{k})=\hbar^{-2} \partial^{2} \varepsilon_{n, \mathbf{k}} / \partial k_{i} \partial k_{j}$ - компоненты тензора обратных эффективных масс.

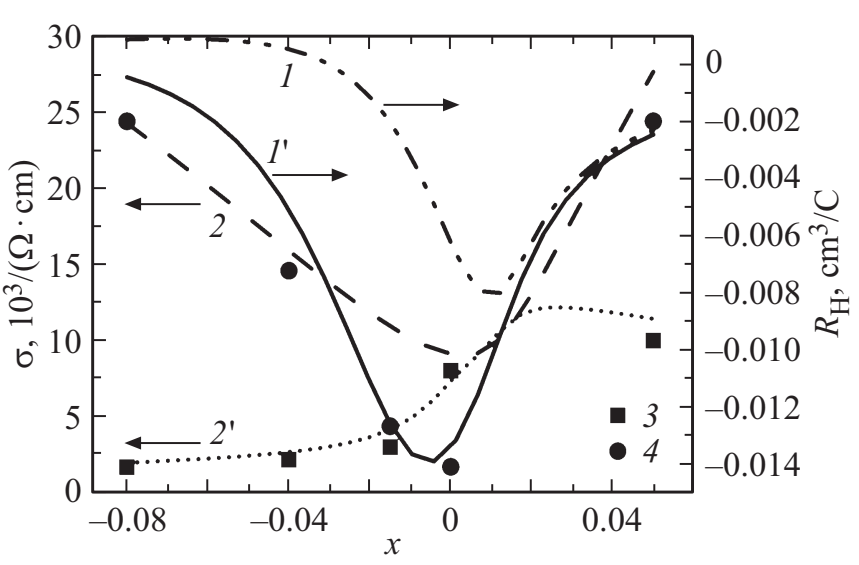

Рис. 4. Концентрационные зависимости $\sigma$ и $R_{\mathrm{H}}$ при комнатной температуре, полученные с использованием первопринципных расчетов электронного спектра в приближении постоянного времени релаксации $\left(1-R_{\mathrm{H}}, 2-\sigma\right)$ и времени релаксации, обратно пропорционального плотности состояний $\left(1^{\prime}-R_{\mathrm{H}}\right.$, $\left.2^{\prime}-\sigma\right)$. Символами обозначены экспериментальные значения $\left(3-\sigma, 4-R_{\mathrm{H}}\right)$.

На рис. 1 кривые $1^{\prime}-3^{\prime}$ построены в приближении постоянного времени релаксации. Видно, что описать температурные зависимости кинетических коэффициентов в таком приближении не удается. Согласно расчетам оказывается, что проводимость возрастает с ростом температуры, но в эксперименте это наблюдается лишь в составах с добавлением железа. Зависимость $R_{\mathrm{H}}(T)$ в данном приближении также не совпадает с экспериментальной (рис. 1).

Если учесть энергетическую зависимость времени релаксации в виде (4), то согласование расчетных и экспериментальных кривых улучшается. Следует заметить, что в таком приближении рассчитанная зависимость $R_{\mathrm{H}}(T)$ содержит характерные особенности, описанные выше, которые невозможно получить в рамках других моделей. Вариация параметров $w_{a}$ и $w_{d}$ позволяет получить хорошее согласие расчетных и экспериментальных температурных зависимостей проводимости, но не изменяет зависимости $R_{\mathrm{H}}(T)$. Величина $w_{d} /\left(300 w_{a}\right)$, характеризующая относительную интенсивность рассеяния на точечных дефектах, равна нулю для чистого CoSi, а для состава с добавлением 5 ат\% $\%$ i - единице. С увеличением содержания $\mathrm{Fe} w_{d} /\left(300 w_{a}\right)$ растет: 1.33 для образца с содержанием $1.5 \mathrm{aT} \% \mathrm{Fe}, 4.5$ - для 4 aт\% $\mathrm{Fe}$ и 7 - для 8 aт\% Fe. Сравнение этих параметров для близких концентраций примесей ( $5 \mathrm{aT} \% \mathrm{Ni}$ и $4 \mathrm{aT} \% \mathrm{Fe})$ показывает, что относительный вклад сплавного рассеяния оказывается выше в образцах, легированных железом. Полученные зависимости показаны на рис. 2.

На рис. 4 приведены концентрационные зависимости $\sigma$ и $R_{\mathrm{H}}$ твердых растворов $\mathrm{Co}_{1-x} \mathrm{Fe}(\mathrm{Ni})_{x} \mathrm{Si}$ при комнатной температуре (отрицательные значения $x$ соответствуют содержанию $\mathrm{Fe}$, положительные - содержанию Ni). Видно, что для всех исследованных составов использование времени релаксации, обратно пропорци- 
онального плотности состояний, позволяет лучше описать кинетические коэффициенты по сравнению с часто используемым в первопринципных расчетах приближением постоянного времени релаксации. Из рис. 3 видно, что плотность состояний резко возрастает в области энергий, лежащих ниже уровня Ферми $\varepsilon_{\mathrm{F}}$ в чистом силициде кобальта. Поэтому подвижность носителей при $\varepsilon<\varepsilon_{\mathrm{F}}$ будет значительно ниже, чем при $\varepsilon>\varepsilon_{\mathrm{F}}$. При легировании никелем (железом) уровень химического потенциала сдвигается вверх (вниз) по отношению к $\varepsilon_{\mathrm{F}}$ в $\mathrm{CoSi}$, в область состояний с большей (меньшей) подвижностью. Наряду с большей интенсивностью примесного рассеяния в образцах с железом это объясняет асимметрию проводимости при легировании никелем и железом.

\section{4. Заключение}

В работе представлены экспериментальные данные о температурных зависимостях коэффициента Холла и проводимости моносилицида кобальта $\mathrm{CoSi}$ и соединений на его основе при температурах 77-800 K. Показано, что в рамках традиционной для полуметаллов модели невозможно описать поведение кинетических коэффициентов во всем температурном диапазоне. Кроме того, параметры, описывающие зонную структуру, механизмы рассеяния и концентрацию носителей, взаимосвязаны и поэтому определяются неоднозначно.

Отчасти такую неоднозначность удается устранить, используя результаты первопринципных расчетов электронного спектра, которые позволяют учесть особенности реальной зонной структуры материала при вычислении кинетических коэффициентов. Однако расчеты с применением наиболее часто используемого в литературе приближения постоянного времени релаксации плохо согласуются с экспериментальными температурными зависимостями.

Модель с использованием времени релаксации, обратно пропорционального полной плотности состояний, более точно описывает зависимость $\sigma(T)$, в том числе и в области низких температур, а также асимметрию зависимости проводимости от состава. Хорошее согласие теории с экспериментом свидетельствует о том, что в данных материалах важную роль играет межзонное рассеяние. Теоретическая зависимость $R_{\mathrm{H}}(T)$ не содержит подгоночных параметров и хуже согласуется с экспериментальной температурной зависимостью. Однако модель неплохо описывает зависимость коэффициента Холла от состава при комнатной температуре и качественно согласуется с полученными температурными зависимостями для образцов разного состава.

\section{Список литературы}

[1] M.I. Fedorov, V.K. Zaitsev. CRC Handbook of thermoelectrics (1995) p. 27.1.

[2] S. Asanabe, D. Shinoda, Y. Sasaki. Phys. Rev., 134, 774 (1964).
[3] A. Sakai, F. Ishii, Y. Onose, T. Yasuhide, Y. Satoshi, A. Hideaki, N. Naoto, T. Yoshinori. J. Phys. Soc. Jpn, 76, 093601 (2007).

[4] D.A. Pshenay-Severin, Y.V. Ivanov, A.T. Burkov, S.V. Novikov, V.K. Zaitsev, H. Reith. J. Electron. Mater. v. 47, 3277 (2017). URL https://doi.org/10.1007/s11664-017-6005-8

[5] D.A. Pshenay-Severin, Y.V. Ivanov, A.A. Burkov, A.T. Burkov. J. Phys.: Condens. Matter, 30, 135501 (2018).

[6] M. Vedernikov, P. Konstantinov, A. Burkov. Proceedings of the Eighth International Conference on Thermoelectric Energy Conversion (Nancy, France, 1989) p. 45.

[7] М.И. Федоров. Автореф. канд. дис. (01.04.10) (Л., 1981).

[8] P. Giannozzi, O. Andreussi, T. Brumme, O. Bunau, M. Buongiorno Nardelli et al. J. Phys.: Condens. Matter, 29, 465901 (2017).

[9] G. Pizzi, D. Volja, B. Kozinsky, M. Fornari, N.Marzari. Comput. Phys. Commun., 185, 422 (2014).

[10] Б.М. Аскеров. Электронные явления переноса в полупроводниках (М., Наука, 1985)

Редактор А.Н. Смирнов

\section{Galvanomagnetic properties of cobalt monosilicide and alloys based on it}

\author{
A.Yu. Ovchinnikov ${ }^{1,2}$, P.P. Konstantinov ${ }^{1}$, \\ D.A. Pshenay-Severin ${ }^{1,2}$, A.T. Burkov ${ }^{1}$ \\ ${ }^{1}$ loffe Institute, \\ 194021 St. Petersburg, Russia \\ ${ }^{2}$ Peter the Great St. Petersburg Polytechnic University, \\ 195251 St. Petersburg, Russia
}

Abstract In this work, we study the Hall coefficient and the conductivity of cobalt monosilide $\mathrm{CoSi}$, as well as $\mathrm{Co}_{1-x} \mathrm{Fe}_{x} \mathrm{Si}$ and $\mathrm{Co}_{1-x} \mathrm{Ni}_{x} \mathrm{Co}$ alloys with contents up to 8 at\% of iron and up to $5 \mathrm{at} \%$ of nickel. The temperature dependences of the Hall coefficient and of the conductivity were measured in the temperature range of $77-800 \mathrm{~K}$. The theoretical interpretation of the experimental dependencies is based on two different models of the electronic structure of the compound: a simple 2-band semimetallic structure with small overlap of isotropic parabolic bands; and $a b$ initio electronic structure, containing near Fermi energy topological features with multiply degenerate intersections of bands. 